\title{
TRIGGERED STARBURSTS IN GALAXY MERGERS
}

\author{
Y. TANIGUCHI, Y. SHIOYA AND T. MURAYAMA \\ Astronomical Institute, Tohoku University \\ Aramaki, Aoba, Sendai 980-77, JAPAN \\ AND \\ K. WADA \\ National Astronomical Observatory of Japan \\ Osawa 2-21-1, Mitaka 181, JAPAN
}

\begin{abstract}
A unified formation mechanism of nuclear starbursts is presented; all the nuclear starbursts are triggered by binary supermassive black holes made in the final phase of galaxy mergers. Minor mergers cause both nuclear starbursts and hot-spot nuclei while major mergers cause (ultra)luminous infrared galaxies. We discuss the case of Arp 220 in detail.
\end{abstract}

\section{Introduction}

It has been considered that nuclear starbursts are driven by gas fueling toward the nuclear regions either by bar structure or by galaxy-galaxy interactions (Shlosman, Begelman, \& Frank 1990; Barnes \& Hernquist 1992). However, non-barred spirals account for $\simeq 50 \%$ of the starburst galaxies and only $30 \%$ of the starburst galaxies have companion galaxies (Balzano 1983; Keel \& van Soest 1992). It is thus suggested that the presence of a bar or an interacting companion may not be a prime mover of the nuclear starbursts.

Recently, Taniguchi \& Wada (1996, hereafter TW96) proposed that a supermassive black hole binary formed by a merger with a nucleated satellite galaxy triggers intense star formation in the central regions of spiral galaxies (see also Gaskell 1985; Hernquist 1989; Mihos \& Hernquist 1994a; Hernquist \& Mihos 1995). They stressed the importance of definite triggering of the starbursts by the supermassive black hole binary relative to that of gas fueling caused by minor mergers. It is known that (ultra)luminous 
starburst galaxies are associated with major mergers between two gas-rich (nucleated) spiral galaxies (Sanders et al. 1988). Taking both minor and major mergers into account, Taniguchi, Wada, \& Murayama (1996) proposed a new unified formation scenario for nuclear starbursts. The minor merger scenario can also explain a starburst-AGN connection because a minor merger with a nucleated satellite drives circumnuclear starbursts and then leads to gas fueling onto the central engine as the merger proceeds (Taniguchi 1997). In this paper, we discuss the case of the archetypical, ultraluminous starburst galaxy Arp 220.

\section{The Case of Arp 220}

It is widely accepted that the dissipative collapse caused by a merger between two gas-rich galaxies is responsible for the intense nuclear starbursts in the ultraluminous infrared galaxies (ULIGs; Sanders et al. 1988; Kormendy \& Sanders 1992). The most important observational property of the starbursts occurring in the ULIGs is that the diameters of the ionized nebulae are only $\sim 100 \mathrm{pc}$ (Condon et al. 1991) and thus it is expected that a starburst core (i.e., a clusters of massive stars) is resided in the inner 10 pc regions in each nucleus. Although a major merger between two gas-rich galaxies can cause the efficient gas fueling into the central region of the merger system, such a gas system would fragment into several gas clumps because of the effect of self gravity (Shlosman \& Noguchi 1993). Therefore the gravitational instability scenario may not explain the compact nature of the starbursts in ULIGs straightforwardly.

Here let us go back to the TW96 model. In order to generate one compact starburst region, the TW96 model needs a pair of supermassive black holes orbiting each other. Therefore, the presence of two compact starburst regions requires two merging nuclei, each of which contains a couple of nuclei (a supermassive black hole binary); i.e., there are four nuclei in total in the double-nucleus ULIGs. If this is the case, Arp 220 would come from a merger among several galaxies (a multiple merger) rather than from a merger between two galaxies. Since there are many compact groups of galaxies (e.g., Hickson 1982), it is not unlikely that some such groups have already merged into their remnants like ULIGs or elliptical galaxies because their merging timescale is generally shorter than the Hubble time (Barnes 1989; Weil \& Hernquist 1996). Another merit of this scenario is that the TW96 model explains both the nuclear compact starbursts and the surrounding poststarburst, as observed in Arp 220 (Larkin et al. 1995), because the starburst regions move to the inner region as the supermassive black hole binary shrinks.

Finally, we mention that there is strong evidence for the multiple merger 
for Arp 220; the VLBI mapping of $\mathrm{OH}$ megamaser emission of Arp 220 shows that there are at least three bright $\mathrm{OH}$ megamaser spots in the nuclear region (Diamond et al. 1989). The eastern nucleus contains the two bright $\mathrm{OH}$ maser spots with a projected separation of $47.6 \mathrm{pc}$ while the western one has only one component. Lonsdale et al. (1994) made a new VLBI measurement of $\mathrm{OH}$ emission of Arp 220 and found that the western (i.e., the brightest) component of the $\mathrm{OH}$ maser originates from a very compact region whose size is less than $1 \mathrm{pc}$. This measurement suggests strongly that the western component is attributed to pumping by far-infrared continuum emitted by a dusty torus around an active galactic nucleus rather than to that by the luminous starburst (see also, however, Skinner et al. 1997). If this is also the case for the two eastern $\mathrm{OH}$ maser components, Arp 220 contains three active galactic nuclei (i.e., three supermassive black holes) at least as suggested by Diamond et al. (1989). Though the western nucleus contains only one $\mathrm{OH}$ maser source, we may consider that the two nuclei aligns accidentally on our line of sight and thus they are not resolved spatially (see Fig. 1). In order to understand what happens in Arp 220 more precisely, we need a next generation millimeter/submillimeter arrays at Atacama.

\section{References}

Balzano, V.A. 1983, $A p J, \mathbf{2 6 8}, 602$

Baan, W.A., \& Haschick, A.D. 1995, ApJ, 454, 745

Barnes, J.E. 1989, Nature, 338, 123

Barnes, J. E., \& Hernquist, L. 1992, ARA \& $A$, 30, 705

Condon, J.J., Huang, Z.-P., Yin, Q.F., \& Thuan, T.X. 1991, ApJ, 378, 65

Diamond, P.J., Norris, R.P., Baan, W.A., \& Booth, R.S. 1989, ApJ, 340, L49

Gaskell, C.M. 1985, Nature, 315, 386

Hernquist, L. 1989, Nature, 340, 687

Hernquist, L., \& Mihos, J.C. 1995, ApJ, 448, 41

Hickson, P. 1982, ApJ, 255, 382

Keel, W.C., \& van Soest, E.T.M. 1992, $A \& A S, \mathbf{9 4}, 553$

Kormendy, J., \& Sanders, D. B. 1992, ApJ, 390, L53

Larkin, J.E., Armus, L., Knop, K., Matthews, K., \& Soifer, B.T. 1995, ApJ, 452, 599

Lonsdale, C.J., Diamond, P.J., Smith, H.E., \& Lonsdale, C.J. 1994, Nature, 370, 117

Mihos, J.C., \& Hernquist, L. 1994, ApJ, 425, L31

Sanders, D.B., et al. 1988, $A p J, \mathbf{3 2 5}, 74$

Shlosman, I., Begelman, M.C., \& Frank, J. 1990, Nature, 345, 679

Shlosman, I., \& Noguchi, M. 1991, ApJ, 414, 474

Skinner, C.J., Smith, H.A., Sturm, E., Barlow, M.J., Cohen, R.J., \& Stacey, G.J. 1997, Nature, 386, 472

Taniguchi, Y. 1997, ApJ, 487, L17

Taniguchi, Y., \& Wada, K. 1996, ApJ, 469, 581

Taniguchi, Y., Wada, K., \& Murayama, T. 1996, Rev. Mex. de A \& A, 6, 240

Weil, M.L., \& Hernquist, L. 1996, ApJ, 460, 101 


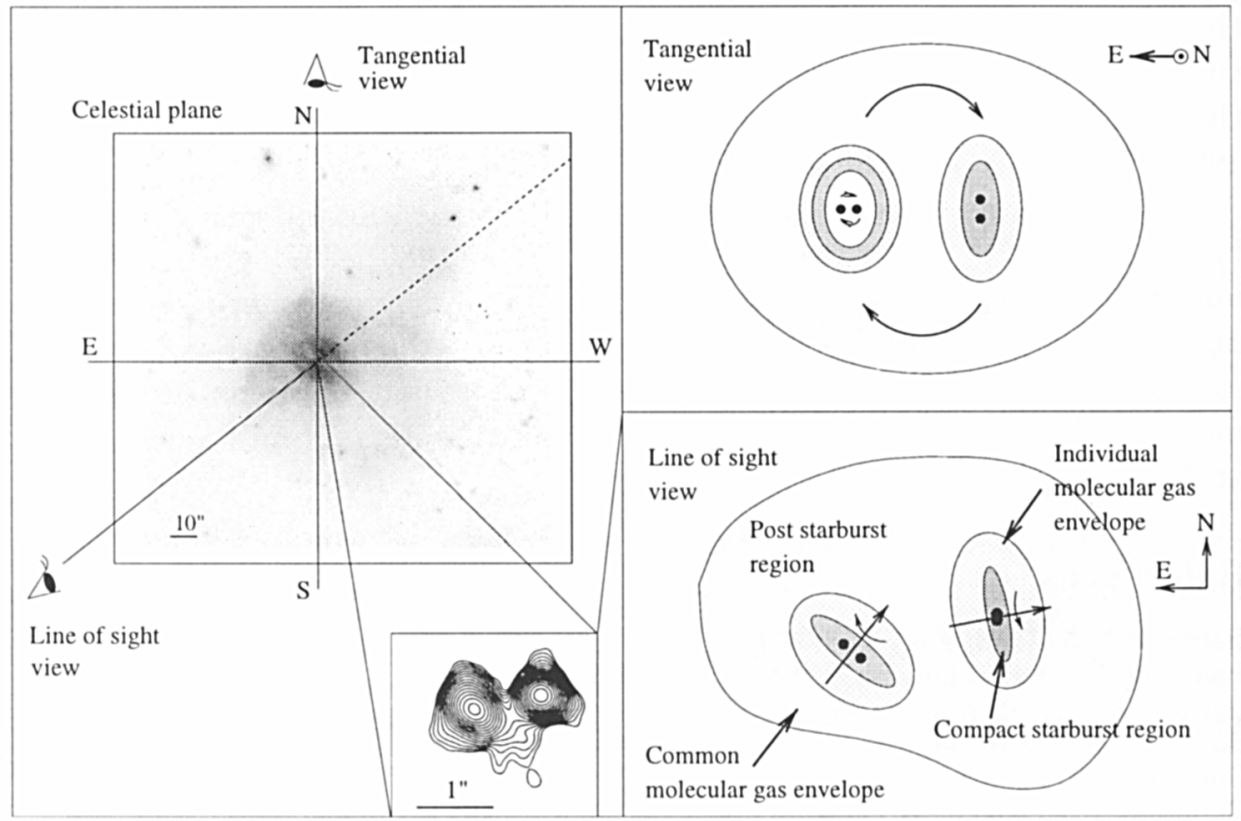

Figure 1. A schematic picture of the multiple merger model of Arp 220. The direct R-band CCD image shown in the left panel is supplied kindly from J. Hibbard \& D. Sanders. The two compact starbursts traced by the the radio continuum imaging at 8.44 $\mathrm{GHz}$ (Condon et al. 1991) are shown in the lower middle panel. The two right panels show the tangential and the line-of-sight view of Arp 220 based on the multiple merger model. The rotation of the eastern black hole binary is determined from the velocity difference between the two $\mathrm{OH}$ megamaser components, IIa and IIb (Diamond et al. 1989) while that of the eastern one is from the rotation of the $\mathrm{H}_{2} \mathrm{CO}$ masing gas (Baan \& Haschick 1995). The global rotation of the $\mathrm{E}$ and $\mathrm{W}$ nuclei is from the NIR spectroscopy (Larkin et al. 1995). 